\title{
Ultra-Compact Dwarf Galaxies - More Massive than Allowed?
}

\author{
Michael Hilker ${ }^{1}$, S. Mieske ${ }^{1}$, H. Baumgardt ${ }^{2}$ and J. Dabringhausen ${ }^{2}$ \\ ${ }^{1}$ ESO, Karl-Schwarzschild-Str. 2, 85748 Garching bei München, Germany \\ email: mhilker@eso.org,smieske@eso.org \\ ${ }^{2}$ AIfA, Universität Bonn, Auf dem Hügel 71, 53121 Bonn, Germany \\ email: holger@astro.uni-bonn.de, joedab@astro.uni-bonn.de
}

\begin{abstract}
Dynamical mass estimates of ultra-compact dwarfs galaxies and massive globular clusters in the Fornax and Virgo clusters and around the giant elliptical Cen A have revealed some surprising results: 1$)$ above $\sim 10^{6} M_{\odot}$ the mass-to-light $(M / L)$ ratio increases with the objects' mass; 2) some UCDs/massive GCs show high $M / L$ values (4 to 6 ) that are not compatible with standard stellar population models; and 3) in the luminosity-velocity dispersion diagram, UCDs deviate from the well-defined relation of "normal" GCs, being more in line with the Faber-Jackson relation of early-type galaxies. In this contribution, we present the observational evidences for high mass-to-light ratios of UCDs and discuss possible explanations for them.
\end{abstract}

Keywords. galaxies: star clusters, galaxies: dwarf, galaxies: kinematics and dynamics

\section{Introduction}

The so-called ultra-compact dwarf galaxies (UCDs) are very massive $\left(10^{6} M_{\odot}<M<\right.$ $\left.10^{8} M_{\odot}\right)$, old, compact stellar systems that were discovered in nearby galaxy clusters about a decade ago (Hilker et al. 1999, Drinkwater et al. 2000). Their nature is unknown yet. Maybe they are remnant nuclei of disrupted galaxies, or maybe they are merged stellar super-clusters formed in interacting galaxies. Regardless of what UCDs actually are, some properties divide them from "ordinary" globular clusters (GCs). The half-light radii of UCDs scale with luminosity reaching $\sim 90 \mathrm{pc}$ for the most massive UCDs. Unlike GCs, their densities within the half-light radii are not increasing with mass but stay at a constant level or even decrease. Thus UCDs are not that compact at all when compared to $10^{6} M_{\odot}$ GCs, but certainly much denser than dwarf ellipticals of comparable mass.

\section{Mass determinations and results}

To estimate the masses of UCDs a new modeling program has been developed that allows a choice of different representations of the surface brightness profile (i.e. Nuker, Sersic or King laws) and corrects the observed velocity dispersions for observational parameters (i.e. seeing, slit size). The derived dynamical masses are compared to those expected from stellar population models. For more details, see Hilker et al. (2007).

The masses, central densities and mass-to-light $(M / L)$ ratios of different hot stellar systems (GCs, UCDs, dEs, bulges and ellipticals) were compared with each other (Dabringhausen et al. 2007, in prep.). The findings are as follows: 1) In the central density vs. mass plane, there seems to be an upper limit of about $10^{4} M_{\odot} / p c^{3}$ for GCs of $\sim 10^{6} M_{\odot}$. UCDs scatter towards lower densities with increasing mass. 2) In the $M / L$ vs. mass plane, the $M / L$ ratio increases with mass above $\sim 10^{6} M_{\odot}$ (see Fig. 1 , right panel), reaching values typical for bulges and ellipticals. 3) When plotting a normalised $M / L$ 

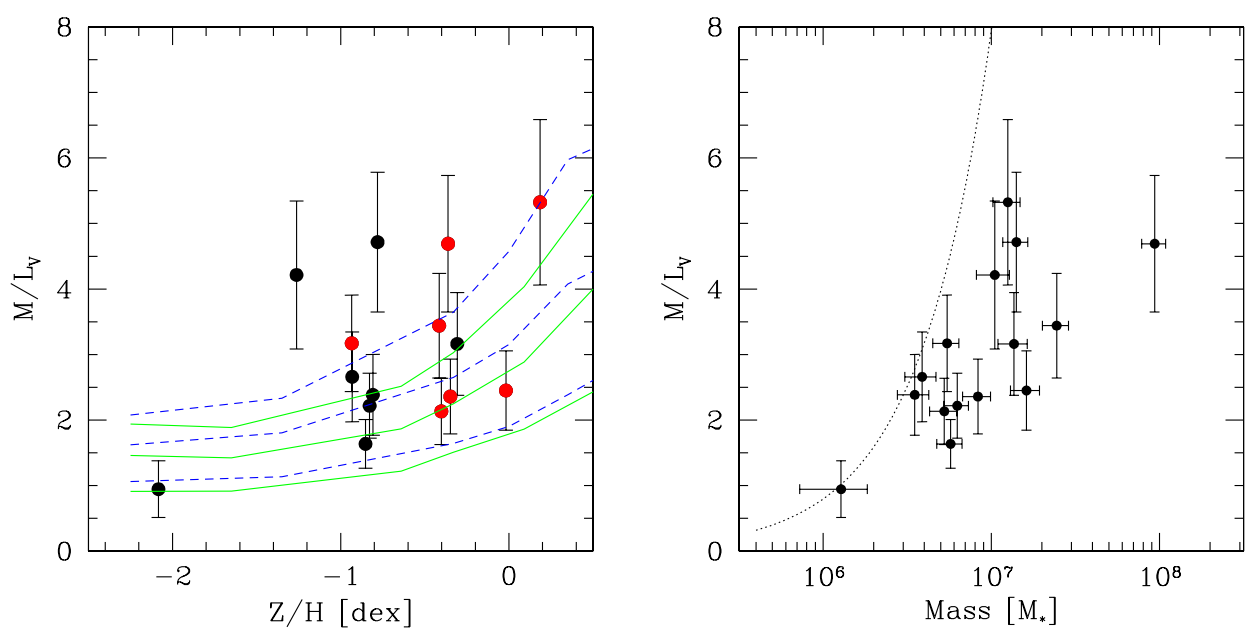

Figure 1. Most recent $M / L_{V}$ determinations for Fornax UCDs from FLAMES/UVES observations (Mieske et al. 2008). Left: SSP models (5, 9 and 13 Gyr) from Bruzual \& Charlot (2003, solid) and Maraston (2005, dashed). Right: The dotted line indicates the observational limit.

ratio (taking out the metallicity dependence on $M / L$ ) vs. mass, the objects more massive than a few times $10^{6} M_{\odot}$ show systematically higher $M / L$ values than the lower mass 'normal' GCs. These high values cannot easily be explained with standard single stellar population models (see Fig. 1, right panel). Interestingly, the transition from low- $M / L$ to high- $M / L$ objects corresponds to the mass regime $\left(10^{6}-10^{7} M_{\odot}\right)$ where the relaxation time at the half-light radius exceeds a Hubble-time.

\section{Possible explanations for unusually high $\mathrm{M} / \mathrm{L}$ ratios}

1) Dark matter: This would imply a very high DM density within the core radius of UCDs. A cuspy NFW halo with $10^{8}-10^{12} M_{\odot}$ would be needed. Might UCDs be surviving dense low mass DM sub-structures?

2) Tidal heating: UCDs might be out of dynamical equilibrium (Fellhauer \& Kroupa 2006). However, very eccentric orbits would be needed to observe high $M / L$-UCDs.

3) Top-heavy IMF: Remnants of massive stars (stellar BHs, neutron stars and white dwarfs) might contribute to the unseen mass and increase the $M / L$ value. An IMF slope of $\alpha=-1$ to -1.5 for $M>1 M_{\odot}$ would be needed.

4) Bottom-heavy IMF: Many low mass stars might contribute to the high $M / L$ value ( $\alpha=-2.35$ low mass slope might explain it).

For a detailed discussion of these points, see Dabringhausen et al. (2007, in prep.) and Mieske et al. (2007, in prep.).

\section{References}

Bruzual, G. A., Charlot, S. 2003, MNRAS 344, 1000

Drinkwater, M. J., Jones, J. B., Gregg, M. D., Phillipps, S. 2000, PASA 17, 227

Fellhauer, M., Kroupa, P. 2006, MNRAS 367, 1577

Hilker, M., Baumgardt, H., Infante, L., et al. 2007, A\&A 463, 777

Hilker, M., Infante, L., Vieira, G., Kissler-Patig, M., Richtler, T. 1999, A\&AS 134, 75

Maraston, C. 2005, MNRAS 362, 799 